\title{
Synergistic protective effects of mizoribine and angiotensin II receptor blockade on cyclosporine A nephropathy in rats
}

Amane Endo', Tomonosuke Someya', Mayu Nakagawa'1, Yayoi Murano'1, Koji Sakuraya' ', Satoshi Hara', Shuichiro Fujinaga², Yoshiyuki Ohtomo', Hitohiko Murakami ${ }^{3}$ and Toshiaki Shimizu'

BACKGROUND: Chronic cyclosporine A (CSA) nephrotoxicity is manifested by renal dysfunction, progressive histopathological kidney lesions characterized by afferent arteriolopathy, and tubulointerstitial fibrosis. In addition to the direct toxic effect of CSA, many other etiological factors such as angiotensin II, transforming growth factor (TGF)- $\beta$, and macrophage infiltration are involved in this pathogenesis. This study investigated the hypothesis that concomitant administration of mizoribine (MZR) and angiotensin II receptor blockade (ARB) may prevent CsA nephrotoxicity in rats.

METHODS: Sprague-Dawley male rats were divided into the following seven groups: group 1, treated with CsA; group 2, treated with CSA + MZR; group 3, treated with CsA + valsartan (Val); group 4, treated with CsA + MZR + Val; group 5, treated with MZR; group 6, treated with Val; and group 7, controls ( $n=5$ each). Renal histopathology and the effect of CsA-induced nephrotoxicity on inflammatory mediators were evaluated.

RESULTS: Results of this study demonstrated that ARB administration significantly decreased arteriolopathy and that in comparison with monotherapy, concomitant administration of MZR and ARB further decreased arteriolopathy, fibrosis, macrophage infiltration, and TGF- $\beta 1$ mRNA expression associated with CSA nephrotoxicity.

CONCLUSION: These findings indicate that MZR and ARB combination treatment provides synergistic protective effects against chronic CSA nephrotoxicity.

yclosporine A (CsA), a fungal cyclic polypeptide, is an immunosuppressant widely used in cases of organ transplantation and autoimmune diseases. CsA administration has been extended to the treatment of nephrotic syndrome in children, particularly in those with steroid dependency and resistant nephrotic syndrome due to steroid toxicity (1). However, the therapeutic benefits of CsA are often limited by chronic nephrotoxicity developing from long-term use of this drug. Chronic CsA nephrotoxicity is manifested by renal dysfunction and progressive histopathological kidney lesions characterized by afferent arteriolopathy, tubular vacuolization, tubular atrophy, and interstitial fibrosis (2).
Mizoribine (MZR) is a purine nucleotide analog isolated from Eupenicillium brefeldianum (3) and has been clinically used as an immunosuppressant following renal transplantation and for lupus nephritis and for nephrotic syndrome. Recent studies have demonstrated that MZR suppresses infiltration of macrophages, which play an important role in the development of interstitial fibrosis and obstructive nephropathy in rats and proliferation of rat glomerular epithelial cells $(4,5)$. Recent reports stated that concomitant administration of MZR significantly improved CsA-induced interstitial fibrosis and macrophage infiltration in CsA-treated rats. However, no decrease in arteriolopathy or expression of transforming growth factor (TGF)- $\beta 1$ was observed after additional MZR administration in a model of CsA nephropathy (6).

On the other hand, activation of the renin-angiotensin-aldosterone system (RAS) influences the pathogenesis of chronic CsA nephropathy $(7,8)$. Several studies have proved that blocking the RAS with either angiotensin-converting enzyme inhibitors or an angiotensin II receptor blockade (ARB) ameliorates arteriolopathy and slows the decline of chronic renal allograft dysfunction with chronic CsA nephropathy $(9,10)$.

Based on these reports, we hypothesize that concomitant administration of MZR and ARB may prevent chronic CsA nephrotoxicity. In this study, the synergistic protective effect of MZR and ARB on the development of CsA nephropathy was investigated.

\section{RESULTS}

\section{Body Weight and Biochemical Analysis}

Body weight and values for blood creatinine/blood urea nitrogen are summarized in Table 1 . At week 4, mean body weight of the rats in the groups treated with CsA, MZR, CsA + MZR, CsA + valsartan (Val), and CsA + MZR + Val was significantly lower than that in the controls $(P<0.01)$. The reduced body weight with nondiarrheal anorexia was observed in the CsA-treated group in comparison with the control group. CsA administration significantly increased the serum creatinine and blood urea nitrogen levels $(P<0.01)$. No improvement in the creatinine and blood urea nitrogen levels was observed with the addition of MZR or Val, but concomitant 
administration of MZR and Val improved levels of these substances in comparison with those in the CsA group $(P<0.01)$. Diarrhea was observed in two rats in the CsA + MZR-treated group, one rat in the CsA + MZR + Val-treated group and one rat in the MZR-treated group. No deaths occurred during the study period.

\section{Kidney Morphology}

Characteristic morphological findings were observed in the CsA-treated rats. These features were usually evident at $4 \mathrm{wk}$ and were similar to those in chronic human CsA renal lesions. Focal interstitial fibrosis, tubular atrophy, and mononuclear inflammatory cell infiltration were observed. Histological analysis of the CsA-treated rats revealed typical arteriolopathy. Smooth muscle cells in the media of the vas afferens were replaced by periodic acid-Schiff-positive material and visualized as a typical circumferential lesion (Figure 1). Arteriolopathy was significantly higher in the CsA group than that in the control group $(40.4 \pm 3.6$ vs. $0.2 \pm 0.3 ; P<$ 0.01 ; Figure 2). Arteriolopathy significantly decreased with the addition of MZR $(24.6 \pm 1.8)$ or Val $(11 \pm 2.1)$ in comparison with the CsA group $(P<0.01$ for each drug), and concomitant administration of MZR and Val $(3.3 \pm 1.9)$ further decreased arteriolopathy in comparison with each drug alone $(P<0.01)$. A significant increase was evident in the amount of fibrosis in the renal cortex in the CsA group in comparison with the control group $(0.35 \pm 0.02$ vs. $0.002 \pm 0.001 ; P<$ 0.01 ; Figures $3,4 a)$. The addition of MZR $(0.17 \pm 0.03)$ or Val $(0.16 \pm 0.01)$ decreased the extent of fibrosis in comparison with the CsA group $(P<0.01$ for each drug). In addition, concomitant administration of MZR and Val $(0.06 \pm 0.01)$ further decreased the extent of fibrosis in comparison with monotherapy $(P<0.01)$.

\section{Immunohistochemistry of ED-1}

Quantitative analysis revealed that the number of ED-1positive cells significantly increased in the CsA group than that in the control group $(110 \pm 18.4$ vs. $8.8 \pm 1.6 ; P<0.01)$. The addition of MZR $(44.2 \pm 7.4)$ or Val $(65.0 \pm 7.2)$ significantly decreased the number of ED-1-positive cells in comparison with the CsA group $(P<0.01$ for each drug). In addition, concomitant administration of MZR and Val $(24.9 \pm 4.1)$ further decreased the number of ED-1-positive cells in comparison with monotherapy $(P<0.01$; Figure 4 b).

\section{RT-PCR for TGF- $\beta 1$ and OPN}

Increased TGF- $\beta 1$ mRNA expression was noted in the CsA group than that in the control group $(1.77 \pm 0.45$ vs. $0.98 \pm 0.13 ; P<0.01)$; this expression remained unchanged with the addition of MZR $(1.65 \pm 0.42)$ or Val $(1.56 \pm 0.31)$ in comparison with the CsA group. However, a significant decrease in the TGF- $\beta 1$ mRNA expression was observed after concomitant administration of MZR and Val (1.16 \pm 0.27$)$ in comparison with the CsA and monotherapy groups $(P<0.05$; Figure 4c).

Osteopontin (OPN) mRNA expression was increased in the CsA group than that in the control group (13.49 \pm 8.59 vs. $1.19 \pm 0.53 ; P<0.01)$. However, it remained unchanged with

Table 1. Body weight gain and biochemical parameters in all groups

\begin{tabular}{|c|c|c|c|c|c|c|c|}
\hline Clinical parameter & Control & CsA & $\mathrm{Cs} A+M Z R$ & $\mathrm{CsA}+\mathrm{Val}$ & $\mathrm{Cs} A+\mathrm{MZR}+\mathrm{Val}$ & MZR & Val \\
\hline $\mathrm{BW}(\mathrm{g})$ & $348.6 \pm 15.8^{* *}$ & $281.8 \pm 17.3^{*}$ & $263.2 \pm 9.6^{*}$ & $281.4 \pm 14.8^{*}$ & $257.0 \pm 11.5^{*}$ & $320.8 \pm 27.0^{*, * *}$ & $352.2 \pm 19.2^{* *}$ \\
\hline Serum creatinine $(\mathrm{mg} / \mathrm{dl})$ & $0.21 \pm 0.02^{* *}$ & $0.66 \pm 0.13^{*}$ & $0.65 \pm 0.38^{*}$ & $0.54 \pm 0.11^{*}$ & $0.40 \pm 0.08^{* *}$ & $0.26 \pm 0.02^{* *}$ & $0.20 \pm 0.02^{* *}$ \\
\hline
\end{tabular}

BUN, blood urea nitrogen; BW, birth weight; CSA, chronic cyclosporine A; MZR, mizoribine; Val, valsartan.

Data are reported as means \pm SEM.

${ }^{*} P<0.01$ vs. control group. ${ }^{* *} P<0.01$ vs. CsA group.

a

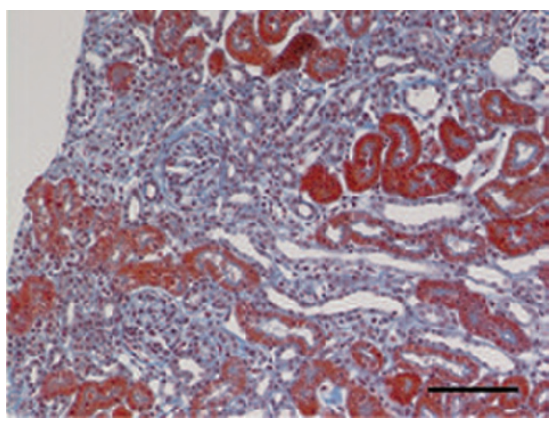

b

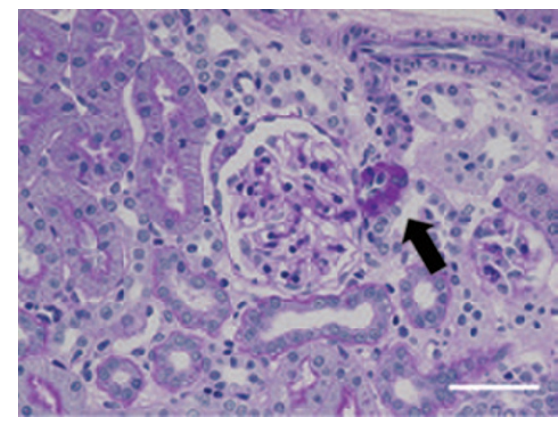

C

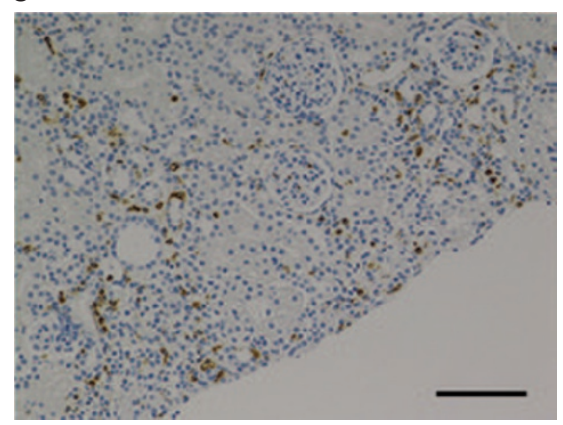

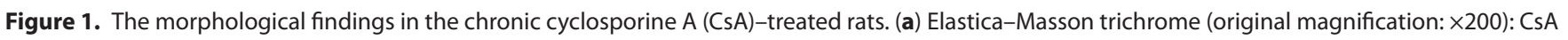
treatment for $4 \mathrm{wk}$ induced typical interstitial fibrosis, inflammatory cell infiltration, and tubular atrophy. Scale bar: $100 \mu \mathrm{m}$. (b) Periodic acid-Schiff (PAS) (original magnification: $\times 400$ ): smooth muscle cells in the media of the vas afferens were replaced by PAS-positive material and visualized as a typical circumferential lesion (black arrow). Scale bar: $50 \mu \mathrm{m}$. (c) Immunohistochemistry of ED-1 (original magnification: $\times 400$ ): ED-1-positive cells were significantly increased in the CsA-treated rats. Scale bar: $100 \mu \mathrm{m}$. 
the addition of MZR $(13.15 \pm 5.87)$ or Val $(10.24 \pm 4.71)$ or MZR + Val (9.24 \pm 4.21$)$ in comparison with the CsA group (Figure 4d).

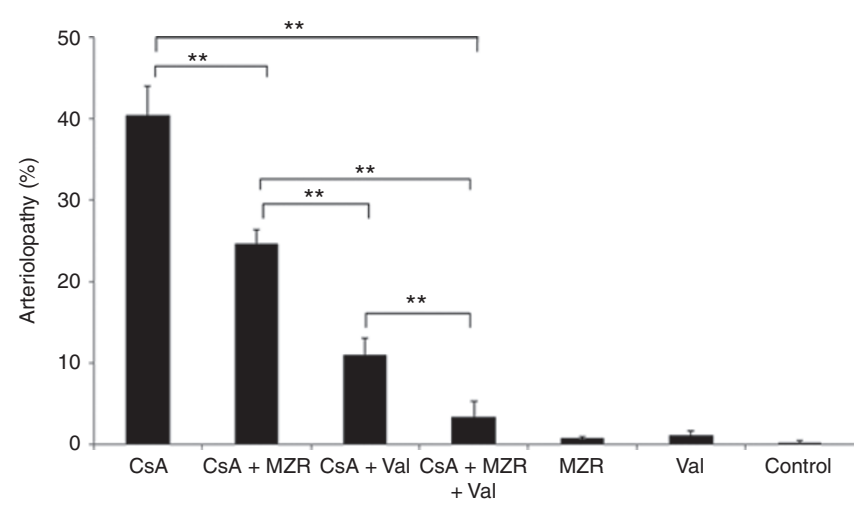

Figure 2. The percentage of arteriolopathy. The quantitatively of arteriolopathy estimated by counting the number of arterioles with arteriolopathy per total number of arterioles available for examination under $\times 200$ magnification. A minimum of 100 arterioles were assessed per biopsy sample. Arteriolopathy was significantly higher in the chronic cyclosporine $A(C s A)$ group than in the control group $\left({ }^{* *} P<0.01\right)$. The addition of mizoribine (MZR) or valsartan (Val) significantly decreased arteriolopathy in comparison with the CsA group $(P<0.01$ for each drug), and concomitant administration of MZR and Val further decreased arteriolopathy in comparison with monotherapy $(P<0.01)$.

\section{Correlations}

Significant positive correlations were found between area of fibrosis and the number of tubulointerstitial ED-1-positive cells/TGF- $\beta 1(r=0.948 ; r=0.723)$. Moreover, a significant correlation was also found between ED-1-positive cells and TGF- $\beta 1$ mRNA expression $(r=0.712)$ (Figure 5).

\section{DISCUSSION}

In this study, concomitant administration of MZR and ARB significantly improved the histopathological severity and renal dysfunction of chronic CsA-related renal damage more than monotherapy with MZR or ARB. These findings suggest that concomitant administration of MZR and ARB can provide beneficial effects against chronic CsA nephrotoxicity.

In recent years, the safety of tacrolimus is being expected, in particular, for organ transplantation and autoimmune diseases. However, calcineurin inhibitor toxicity remains a very important problem for several renal diseases such as steroid dependent and steroid resistant nephrotic syndrome. Many investigators have attempted to elucidate the pathogenesis of CsA nephropathy. Histopathological characteristics of CsA nephrotoxicity include arteriolopathy and fibrosis. Based on these vascular alternations, several vasoconstrictive mechanisms, including activation of the tissue RAS, have been reported to increase endothelin production, which is a

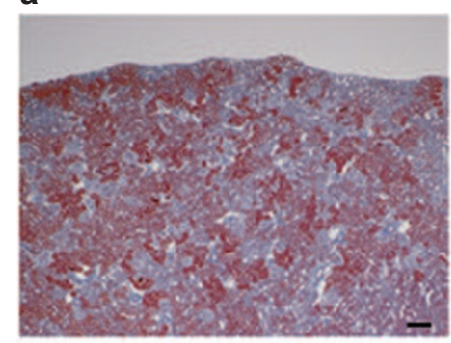

d

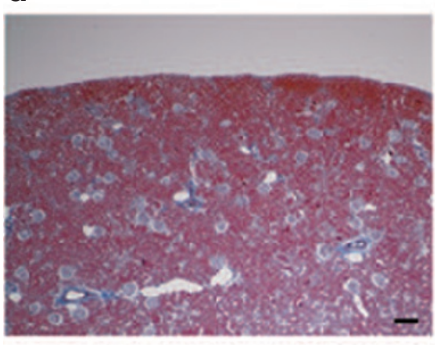

g

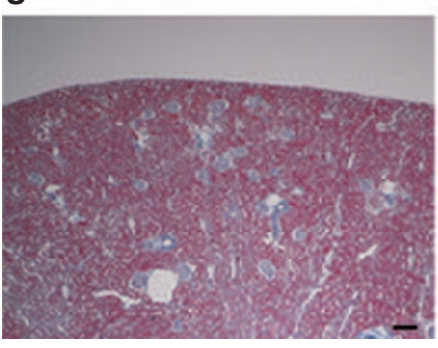

b

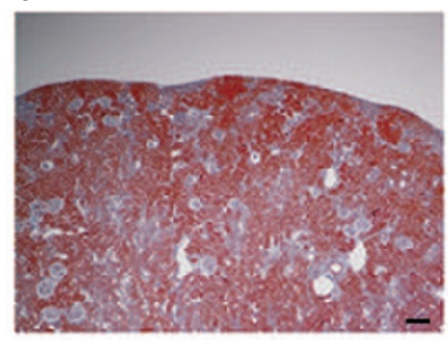

e

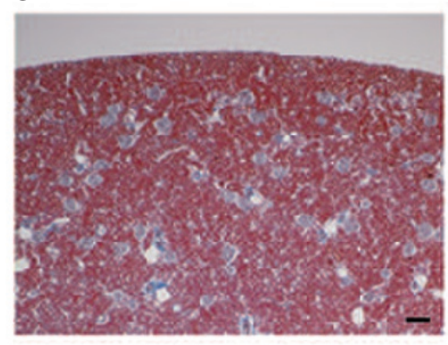

C

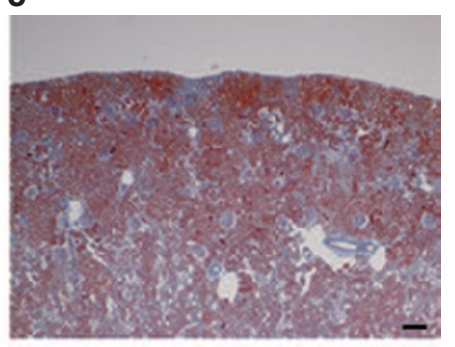

f

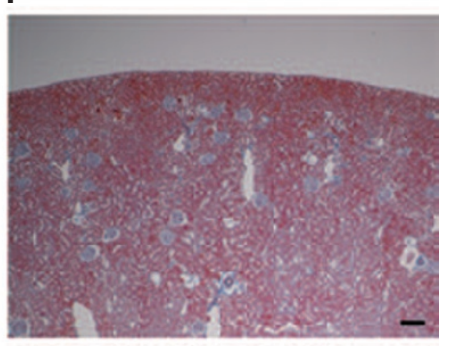

Figure 3. Elastica-Masson trichrome-stained interstitial fibrosis (a) treated with CsA; (b) treated with CsA + MZR; (c) treated with CsA + Val; (d) treated with CsA + MZR + Val; (e) treated with MZR; (f) treated with Val; and (g) controls (original magnification: $\times 10$; scale bar: $200 \mu \mathrm{m}$ ). CsA, chronic cyclosporine A; MZR, mizoribine; Val, valsartan. 
a
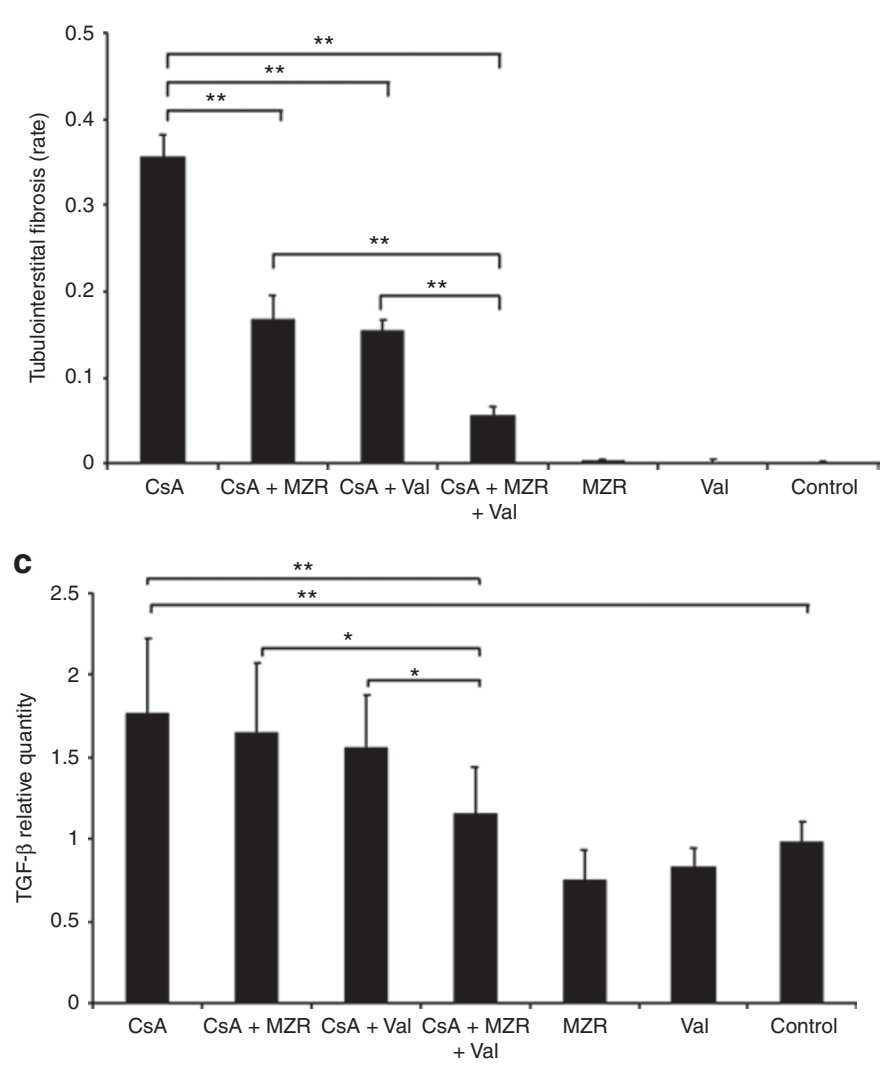

b

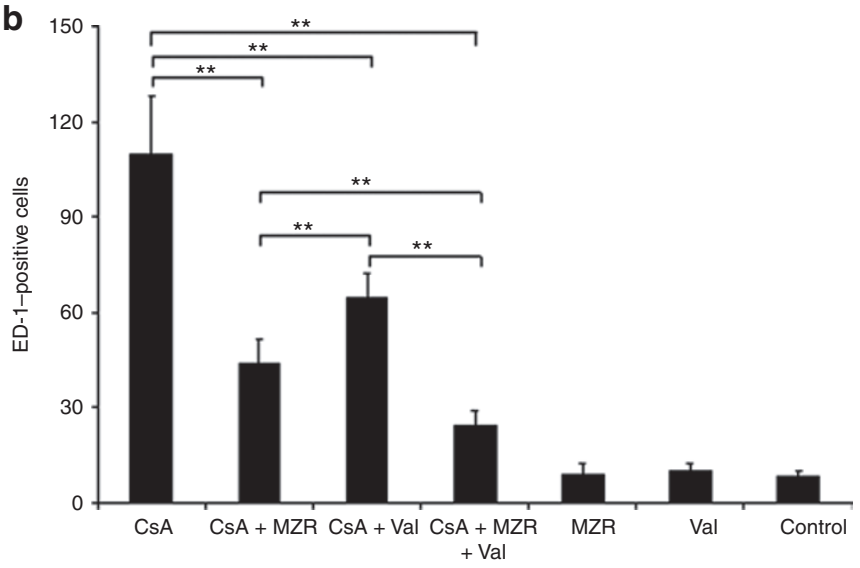

d

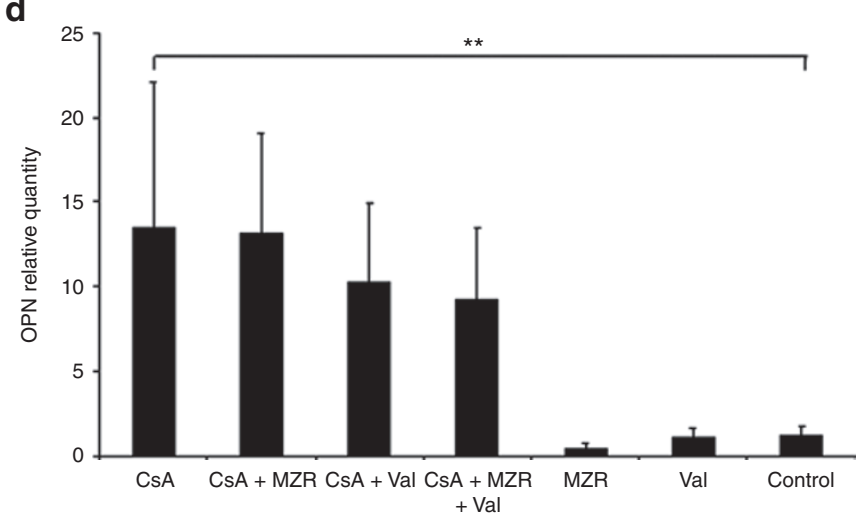

Figure 4. (a) Quantitive analysis on tubulointerstitial fibrosis. The quantification of interstitial fibrosis was evaluated by color image analyzer on blue staining area of elastica-Masson trichrome. The extent of interstitial fibrosis was significantly increased in the renal cortex in the chronic cyclosporine A (CsA) group in comparison with the control group $\left.{ }^{* *} P<0.01\right)$. The addition of mizoribine (MZR) or valsartan $(V a l)$ decreased the extent of fibrosis in comparison with the CsA group $(P<0.01$ for each drug). In addition, concomitant administration of MZR and Val further decreased the extent of fibrosis in comparison with monotherapy $(P<0.01)$. (b) Immunohistochemistry of ED-1. Macrophage infiltration was determined by the number of cells in the cortex that stained positively with the ED-1 antibody. The number of ED-1-positive cells was counted in at least 10 fields of cortex per section under $\times 200$ magnification. A quantitative analysis revealed a significant increase in the number of ED-1-positive cells in the CsA group in comparison with the control group (**P<0.01). The addition of MZR or Val significantly decreased the number of ED-1-positive cells in comparison with the CsA group ( $P<0.01$ for each drug). In addition, concomitant administration of MZR and Val further decreased the number of ED-1-positive cells in comparison with monotherapy $(P<0.01)$. (c) Real-time PCR for TGF- $\beta 1$. In the CsA group, TGF- $\beta 1$ mRNA expression was increased in comparison with the control group ( $\left.{ }^{* *} P<0.01\right)$. No decrease in the TGF- $\beta 1$ mRNA expression was observed with the addition of MZR or Val in comparison with the CsA group, but concomitant administration of MZR and Val significantly decreased TGF$\beta 1$ mRNA expression in comparison with the CsA and monotherapy group ( $\left.{ }^{*} P<0.05\right)$. (d) Real-time PCR for osteopontin (OPN). In the CsA group, OPN mRNA expression was increased in comparison with the control group ( ${ }^{* *} P<0.01$ ). No decrease in the OPN mRNA expression was observed with the addition of MZR or/and Val in comparison with the CsA group.

involved in the development of CsA nephropathy (11-13). Myers et al. (14) were the first to report that CsA not only is associated with reversible alterations in renal vascular resistance but also induces irreversible changes in the renal structure. More detailed histological analyses later demonstrated all three processes of CSA nephrotoxicity-arteriolopathy (afferent arteriolar hyalinosis), tubulointerstitial changes (tubular atrophy and interstitial fibrosis), and glomerular lesions (thickening of Bowman's capsule and focal segmental glomerular sclerosis) $(15,16)$.

In cases of long-term CsA use, afferent arteriolopathy and narrowing of the arteriolar lumen is likely to be a major contributor to the progression of tubulointerstitial fibrosis. Local hypoxia and ischemia of the tubulointerstitial area, resulting from renal vasoconstrictive mechanisms induced by CsA, lead to the formation of free radicals and reactive oxygen species
$(17,18)$. In addition to the direct toxic effect of CsA, many mediators such as angiotensin II, TGF- $\beta$, and OPN are involved in the pathogenesis of CsA nephropathy $(19,20)$. Upregulation of TGF- $\beta$ and inflammation with macrophage infiltration is considered an important etiological factor associated with interstitial striped fibrosis in chronic CsA nephropathy $(21,22)$. In our study, CsA administration in salt-depleted rats resulted in afferent arteriolopathy, interstitial fibrosis, macrophage infiltration, and TGF- $\beta 1 / \mathrm{OPN}$ expression. These findings were consistent with those of previous reports regarding functional and morphological changes in chronic CsA nephrotoxicity.

On the other hand, MZR is a selective inhibitor of inosine monophosphate dehydrogenase, which is the key enzyme in de novo synthesis of purine nucleotides (23). MZR is an immunosuppressant that has a similar effect as that of azathioprine and mycophenolate mofetil, and it has the advantage of very 
a
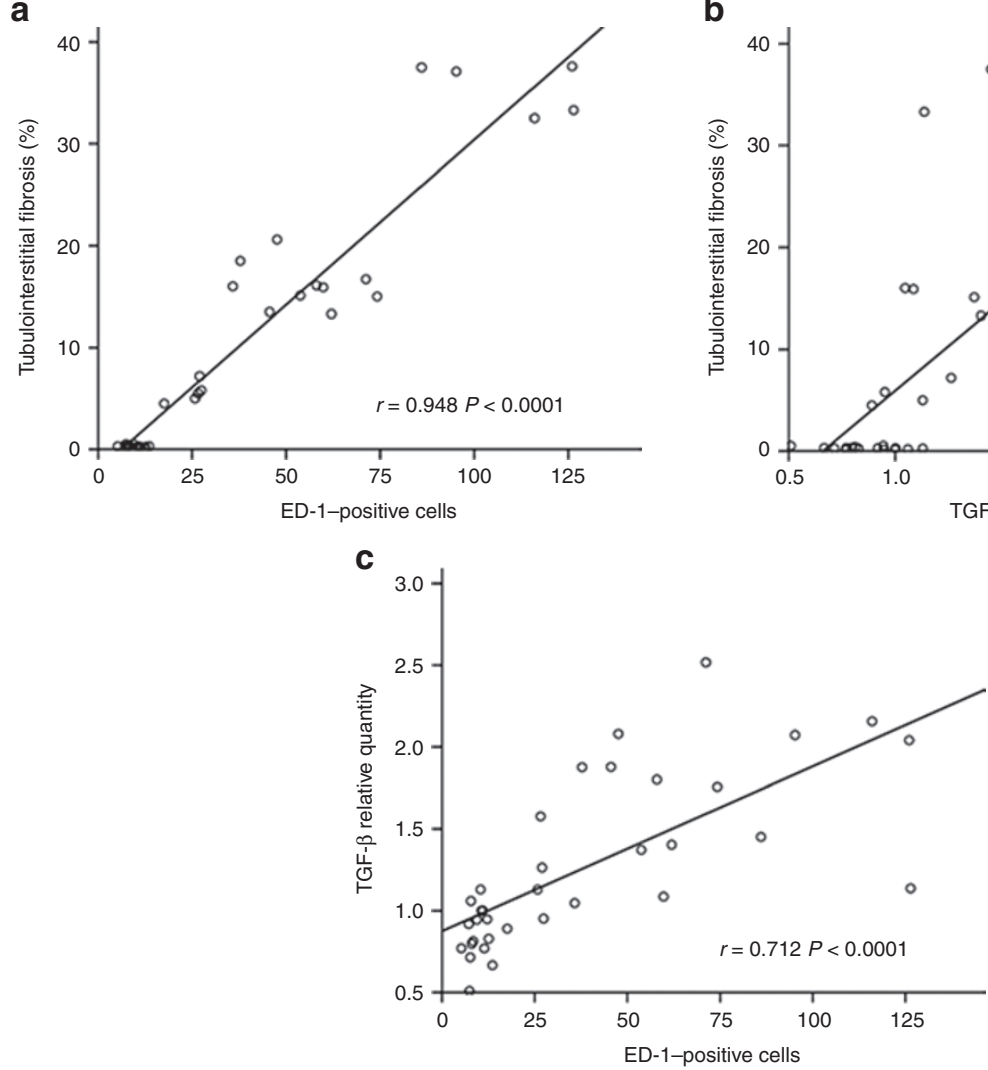

b

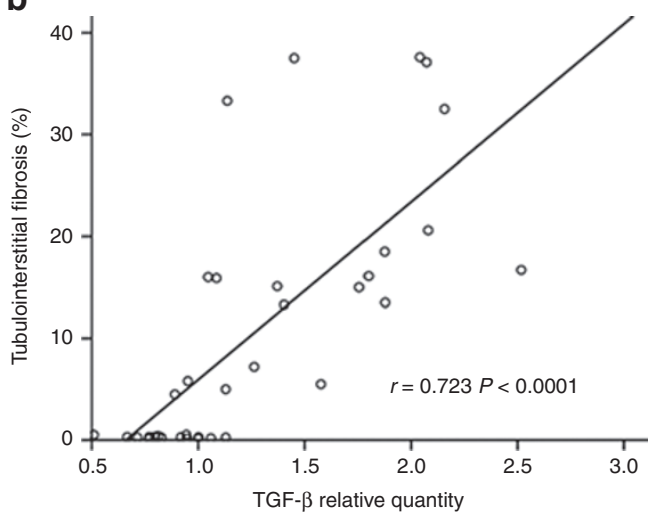

$\beta$ relative quantity

Fetween area of tubulointerstitial fibrosis and the number of ED-1-positive cells $(r=0.948 ; P<0.0001)$. (b) Correlation between area of tubulointerstitial fibrosis and TGF- $\beta 1$ mRNA expression $(r=0.723 ; P<0.0001)$. (c) Correlation between TGF- $\beta 1$ mRNA expression and area of tubulointerstitial fibrosis $(r=0.712, P<0.0001)$. TGF, transforming growth factor.

few side effects even with long-term administration. MZR has been developed in Japan, and it has been used mainly in Asia. In addition to its use after renal transplantation, recent studies have demonstrated the efficacy and safety of MZR for treatment of renal diseases, including lupus nephritis, IgA nephropathy, and childhood nephrotic syndrome (24-26). Moreover, MZR has been reported to decrease macrophage infiltration, OPN secretion, and TGF- $\beta$ expression; this decrease has been associated with improved histopathology in an animal model of unilateral ureteral obstruction (27). Recently, Matsui et al. (28) reported that MZR attenuates inflammatory response and macrophage infiltration against bleomycin-induced lung injury in mice. Recent reports previously indicated significant improvement in interstitial fibrosis and macrophage infiltration but no decrease in arteriolopathy and expression of TGF$\beta 1$ in CsA-treated rats after administration of MZR (6).

Several studies have reported clinical improvement in histological arteriolopathy and renal allograft dysfunction with chronic CsA use as a result of the RAS blockade with either angiotensin-converting enzyme inhibitors or ARB. For example, in an experimental model of chronic CsA nephropathy, ARB was reported to decrease the expression of TGF- $\beta$ and upregulate the expression of Klotho, an antiaging gene $(29,30)$.

On the basis of these previous studies, MZR and/or ARB may have renoprotecitve effects on the development of CsA nephropathy. Our results demonstrated that the addition of
ARB significantly decreased arteriolopathy caused by CsA nephropathy in rats and concomitant administration of MZR, and ARB further decreased arteriolopathy, fibrosis, and TGF$\beta 1$ mRNA expression in comparison with monotherapy. Moreover, comparing the ARB and MZR monotherapy, MZR had more capacity to suppress the number of ED-1-positive cells, and ARB had more capacity to suppress arteriolopathy. The precise mechanisms by which MZR prevented the progression of CsA nephropathy are not known. However, it could suppress the proliferation of macrophages. The positive correlations between the number of interstitial ED-1-positive cells and fibrosis in the kidneys may support this hypothesis. We also found that the protein levels of TGF- $\beta 1$ correlated positively with the degree of fibrosis. These results suggest that ED-1-positive cells might be upregulated via TGF- $\beta 1$ in the kidney and that these may play an important role in tubulointerstitial fibrosis.

These finding suggests that MZR is effective in preventing the inflammatory process and interstitial fibrosis in chronic CsA-induced nephropathy. On the other hand, the inhibition of vascular endothelial disorders and angiotensin II type 2 receptor-mediated signaling effect by the ARB may have suppressed CsA nephropathy in the upstream of the process of inflammation. Activation of the RAS, especially the intrarenal RAS, mediates vasoconstriction hemodynamically and thus leads to low-grade ischemia. Whereas, the RAS may also 
induce renal injury nonhemodynamically via stimulation of tubulointerstitial inflammation, the expression of TGF- $\beta 1 /$ OPN. Blocking this system with ARB mitigates all of these parameters and confers a renoprotective effect.

In our study, the improvement of arteriolopathy indicates that ARB ameriolate hemodynamical RAS activation. Theoretically, ARB suppressed the activation of the RAS, in which angiotensin II, through the activation of AT1 receptors, not only participates in renal vasoconstriction but also promotes fibrotic processes and the release of aldosterone. On the other hand, MZR may suppress influx of interstitial $\mathrm{T}$ lymphocytes by directly inhibiting the proliferation of $\mathrm{T}$ lymphocytes in addition to macrophage attenuation. These additional factors may affect the discrepancy between pathological alternation and TGF- $\beta 1 / O P N$ expression.

Our data suggest that concomitant administration of MZR and ARB has synergistic protective effects. Combined treatment with these agents may have suppressed infiltration of inflammatory cells and inflammatory molecules associated with CsA nephrotoxicity.

In summary, the results of this study demonstrated a protective effect of concomitant MZR and ARB administration and synergistic effects of these agents on chronic CsA nephrotoxicity. These beneficial effects were observed in several processes that contribute to interstitial fibrogenesis. These results suggest that combined treatment with MZR and ARB has the potential for therapeutic use in patients with CsA nephropathy.

\section{METHODS}

\section{Animals}

Male Sprague-Dawley rats weighing 200-250 g were housed in cases in a temperature- and light-controlled environment and fed a lowsalt diet ( $0.03 \%$ sodium; Test Diet, Richmond, IN) at the Juntendo University Animal Care facility. The protocol used in this study was approved by the Juntendo University Animal Care Committee (Tokyo, Japan).

\section{Drugs}

CsA (ProSpec-Tany TechnoGene, East Brunswick, NJ) was diluted in coconut oil (Miglyol 812; Dynamit Nobel AG, Troisdorf, Germany) to a final concentration of $30 \mathrm{mg} / \mathrm{ml}$. MZR (Bredinin; Asahi Chemical Industry, Tokyo, Japan) was prepared in a physiological salt solution to a final concentration of $3 \mathrm{mg} / \mathrm{ml}$. Val (Tokyo Chemical Industry, Tokyo, Japan) was prepared in $0.5 \%$ carboxymethylcellulose sodium (CMC-Na) to a final concentration of $0.5 \mathrm{mg} / \mathrm{ml}$. CsA and MZR were administered intraperitoneally to the rats before precipitation. The solutions were freshly prepared and used on the same day.

\section{Experimental Groups}

The experiment comprised seven groups with five rats each. In the CsA group, the rats received a daily intraperitoneal injection of CsA $(30 \mathrm{mg} / \mathrm{kg})$ and physiological salt solution $(1 \mathrm{ml} / \mathrm{kg})$ as well as $0.5 \%$ CMC-Na $(1 \mathrm{ml} / \mathrm{kg})$ orally for $4 \mathrm{wk}$. In the CsA + MZR group, the rats received a daily intraperitoneal injection of CsA $(30 \mathrm{mg} / \mathrm{kg})$ and MZR $(3 \mathrm{mg} / \mathrm{kg})$ as well as $0.5 \% \mathrm{CMC}-\mathrm{Na}(1 \mathrm{ml} / \mathrm{kg})$ orally for $4 \mathrm{wk}$. In the $\mathrm{CsA}+$ Val group, the rats received a daily intraperitoneal injection of CsA $(30 \mathrm{mg} / \mathrm{kg})$ and physiological salt solution $(1 \mathrm{ml} / \mathrm{kg})$ as well as Val $(0.5 \mathrm{mg} / \mathrm{kg})$ orally for $4 \mathrm{wk}$. In the CsA + MZR + Val group, the rats received a daily intraperitoneal injection of CsA $(30 \mathrm{mg} /$ $\mathrm{kg})$ and MZR $(3 \mathrm{mg} / \mathrm{kg})$ as well as Val $(0.5 \mathrm{mg} / \mathrm{kg})$ orally for $4 \mathrm{wk}$. In the MZR group, the rats received a daily intraperitoneal injection of MZR (3 mg/kg) and Miglyol $812(1 \mathrm{ml} / \mathrm{kg})$ as well as $0.5 \% \mathrm{CMC}-\mathrm{Na}$ $(1 \mathrm{ml} / \mathrm{kg})$ orally for $4 \mathrm{wk}$. In the Val group, the rats received a daily intraperitoneal injection of Miglyol $812(1 \mathrm{ml} / \mathrm{kg})$ and physiological salt solution $(1 \mathrm{ml} / \mathrm{kg})$ as well as Val $(0.5 \mathrm{mg} / \mathrm{kg})$ orally for $4 \mathrm{wk}$. In the control group, the rats received a daily intraperitoneal injection of Miglyol $812(1 \mathrm{ml} / \mathrm{kg})$ and physiological salt solution $(1 \mathrm{ml} / \mathrm{kg})$ as well as $0.5 \% \mathrm{CMC}-\mathrm{Na}(1 \mathrm{ml} / \mathrm{kg})$ orally for $4 \mathrm{wk}$.

\section{Basic Protocol}

Weight-matched rats were randomly assigned to the different treatment groups, and their daily body weight was recorded. At the end of the experiment, blood samples were obtained to evaluate the creatinine, blood urea nitrogen, and whole-blood CsA trough levels. The rats were sacrificed, and their kidneys were removed.

\section{Kidney Morphology}

Tissue samples were fixed in $10 \%$ (vol/vol) formalin, processed by conventional methods, and embedded in paraffin. Sections were stained with hematoxylin-eosin, periodic acid-Schiff, periodic acid silver-methenamine, and elastica-Masson trichrome reagents for evaluation of interstitial lesions and arteriolopathy. Arteriolopathy of afferent arterioles, especially in the vas afferens, was characterized by expansion of the cell cytoplasm of the terminal arteriolar smooth muscle cells by an eosinophilic argentaffin material and quantitatively estimated by counting the number of arterioles with arteriolopathy per total number of arterioles available for examination under $\times 200$ magnification. A minimum of 100 arterioles were assessed per biopsy sample. Interstitial fibrosis was evaluated by blue staining with elastic-Masson trichrome. A color image analyzer (image J 1.46 for Windows; National Institutes of Health, Bethesda, MD) was used for quantification of the percentage of injured areas.

\section{Immunohistochemistry of ED-1}

Infiltrating macrophages were immunohistochemically detected as follows. The deparaffinized sections from tissues fixed with methanolCarnoy's solution were stained using the streptavidin-biotin method. Mouse antirat ED-1 antibody (monocyte/macrophage marker, clone ED-1; AbD Serotec, Kidlington, UK) was used as the primary monoclonal antibody. After treatment for 10 min with $3 \%$ hydrogen peroxide to quench endogenous peroxidase and further treatment for $30 \mathrm{~min}$ with endogenous biotin-blocking agent (DAKO, Glostrup, Denmark), the sections were incubated with the primary antibody at room temperature for $60 \mathrm{~min}$. Animal serum in which the primary antibody had been developed was used as a negative control. Biotinylated goat antimouse immunoglobulin (Ig) G was then used as a secondary antibody for $30 \mathrm{~min}$. Final incubation was performed for $30 \mathrm{~min}$ with peroxidase-streptavidin conjugate, and positive reactions were visualized by a reaction with 3,3'-diaminobenzidine for $5 \mathrm{~min}$. The sections were finally counterstained with Meyer's hematoxylin solution for $1 \mathrm{~min}$. Macrophage infiltration was determined by the number of cells in the cortex that stained positively with the ED-1 antibody. The number of ED-1-positive cells was counted in at least 10 fields of cortex per section under $\times 200$ magnification.

\section{RT-PCR for TGF- $\beta 1$ and OPN}

Real-time polymerase chain reaction (RT-PCR) was performed to detect expression of TGF- $\beta 1$ and OPN in the renal cortex. TaqMan probe-based quantitative RT-PCR was performed using cDNA synthesized from kidney biopsy RNA preparations (High Capacity cDNA Reverse Transcription Kit; Applied Biosystems, Foster City, CA) analyzed using a 7500 Fast Real-Time PCR system (Applied Biosystems) and the default protocols. The expression of each gene was normalized to the expression of glyceraldehyde 3-phosphate dehydrogenase using the standard curve method. Primers and probes for TGF- $\beta 1$ (Rn00572010_m1) and OPN (Rn01449972_m1) were prepared using TaqMan Gene Expression Assays (Applied Biosystems, Technologies, Carlsbad, CA).

\section{Statistical Analyses}

Data are reported as means \pm SEM (SE). Multiple comparisons among groups were performed by one-way ANOVA with the post hoc Bonferroni/Dunn test, and a $P$ value $<0.05$ was considered statistically significant. Linear regression models were used to assess the correlation between two variables. 


\section{ACKNOWLEDGMENTS}

The authors are grateful to Yumiko Sakurai and Takako Ikegami of the Division of Molecular and the Biochemical Research, Biomedical Research Center, Juntendo University Graduate School of Medicine, for their extended technical support.

\section{STATEMENT OF FINANCIAL SUPPORT}

No financial assistance was received to support this study.

\section{REFERENCES}

1. Fujinaga S, Kaneko K, Muto T, Ohtomo Y, Murakami H, Yamashiro Y. Independent risk factors for chronic cyclosporine induced nephropathy in children with nephrotic syndrome. Arch Dis Child 2006;91:666-70.

2. Thomas SE, Andoh TF, Pichler RH, et al. Accelerated apoptosis characterizes cyclosporine-associated interstitial fibrosis. Kidney Int 1998;53:897908.

3. Mizuno K, Tsujino M, Takada M, Hayashi M, Atsumi K. Studies on bredinin. I. Isolation, characterization and biological properties. J Antibiot 1974;27:775-82.

4. Sakai T, Kawamura T, Shirasawa T. Mizoribine improves renal tubulointerstitial fibrosis in unilateral ureteral obstruction (UUO)-treated rat by inhibiting the infiltration of macrophages and the expression of alphasmooth muscle actin. J Urol 1997;158:2316-22.

5. Yamabe H, Shimada M, Murakami R, Fujita T, Shimaya Y, Nakamura N. Mizoribine suppresses proliferation of rat glomerular epithelial cells in culture and inhibits increase of monocyte chemoattractant protein-1 and macrophage inflammatory protein-2 stimulated by thrombin. Biol Pharm Bull 2012;35:705-8.

6. Hara S, Umino D, Someya T, et al. Protective effects of mizoribine on cyclosporine A nephropathy in rats. Pediatr Res 2009;66:524-7.

7. Yang CW, Ahn HJ, Kim WY, et al. Influence of the renin-angiotensin system on epidermal growth factor expression in normal and cyclosporinetreated rat kidney. Kidney Int 2001;60:847-57.

8. Lassila M. Interaction of cyclosporine A and the renin-angiotensin system; new perspectives. Curr Drug Metab 2002;3:61-71.

9. Lin J, Valeri AM, Markowitz GS, D’Agati VD, Cohen DJ, Radhakrishnan J. Angiotensin converting enzyme inhibition in chronic allograft nephropathy. Transplantation 2002;73:783-8.

10. Yang CW, Ahn HJ, Kim WY, et al. Synergistic effects of mycophenolate mofetil and losartan in a model of chronic cyclosporine nephropathy. Transplantation 2003;75:309-15.

11. Müller-Schweinitzer E. Changes in the venous compliance by bradykinin and angiotensin II and its significance for the vascular effects of cyclosporine-A. Naunyn Schmiedebergs Arch Pharmacol 1988;338:699-703.

12. Julien J, Farge D, Kreft-Jais C, et al. Cyclosporine-induced stimulation of the renin-angiotensin system after liver and heart transplantation. Transplantation 1993;56:885-91.

13. Takeda Y, Itoh Y, Yoneda T, Miyamori I, Takeda R. Cyclosporine A induces endothelin-1 release from cultured rat vascular smooth muscle cells. Eur J Pharmacol 1993;233:299-301.
14. Myers BD, Ross J, Newton L, Luetscher J, Perlroth M. Cyclosporine-associated chronic nephropathy. N Engl J Med 1984;311:699-705.

15. Mihatsch MJ, Kyo M, Morozumi K, Yamaguchi Y, Nickeleit V, Ryffel B. The side-effects of ciclosporine-A and tacrolimus. Clin Nephrol 1998;49:356-63.

16. Morozumi K, Takeda A, Uchida K, Mihatsch MJ. Cyclosporine nephrotoxicity: how does it affect renal allograft function and transplant morphology? Transplant Proc 2004;36(2 Suppl):251S-6S.

17. Zhong Z, Arteel GE, Connor HD, et al. Cyclosporin A increases hypoxia and free radical production in rat kidneys: prevention by dietary glycine. Am J Physiol 1998;275(4 Pt 2):F595-604.

18. Djamali A. Oxidative stress as a common pathway to chronic tubulointerstitial injury in kidney allografts. Am J Physiol Renal Physiol 2007;293:F445-55.

19. Pichler RH, Franceschini N, Young BA, et al. Pathogenesis of cyclosporine nephropathy: roles of angiotensin II and osteopontin. J Am Soc Nephrol 1995;6:1186-96.

20. Shihab FS, Andoh TF, Tanner AM, et al. Role of transforming growth factor-beta 1 in experimental chronic cyclosporine nephropathy. Kidney Int 1996;49:1141-51.

21. Islam M, Burke JF Jr, McGowan TA, et al. Effect of anti-transforming growth factor-beta antibodies in cyclosporine-induced renal dysfunction. Kidney Int 2001;59:498-506.

22. Young BA, Burdmann EA, Johnson RJ, et al. Cellular proliferation and macrophage influx precede interstitial fibrosis in cyclosporine nephrotoxicity. Kidney Int 1995;48:439-48.

23. Pankiewicz KW, Patterson SE, Black PL, et al. Cofactor mimics as selective inhibitors of NAD-dependent inosine monophosphate dehydrogenase (IMPDH)-the major therapeutic target. Curr Med Chem 2004;11:887900.

24. Tanaka H, Oki E, Tsuruga K, et al. Mizoribine treatment of young patients with severe lupus nephritis: a clinicopathologic study by the tohoku pediatric study group. Nephron Clin Pract 2008;110:c73-9.

25. Kawasaki Y, Hosoya M, Suzuki J, et al. Efficacy of multidrug therapy combined with mizoribine in children with diffuse IgA nephropathy in comparison with multidrug therapy without mizoribine and with methylprednisolone pulse therapy. Am J Nephrol 2004;24:576-81.

26. Yoshioka K, Ohashi Y, Sakai T, et al. A multicenter trial of mizoribine compared with placebo in children with frequently relapsing nephrotic syndrome. Kidney Int 2000;58:317-24.

27. Sato N, Shiraiwa K, Kai K, et al. Mizoribine ameliorates the tubulointerstitial fibrosis of obstructive nephropathy. Nephron 2001;89:177-85.

28. Matsui K, Ueda H, Terada M, Azuma N, Okamura H, Sano H. Mizoribine protects against bleomycin-induced lung injury. Mod Rheumatol 2010;20:471-7.

29. Shihab FS, Bennett WM, Tanner AM, Andoh TF. Angiotensin II blockade decreases TGF-betal and matrix proteins in cyclosporine nephropathy. Kidney Int 1997;52:660-73.

30. Yoon HE, Ghee JY, Piao S, et al. Angiotensin II blockade upregulates the expression of Klotho, the anti-ageing gene, in an experimental model of chronic cyclosporine nephropathy. Nephrol Dial Transplant 2011;26:800-13. 